\title{
Preoperative considerations, operative preparation, and postoperative care for rib cartilage use in rhinoplasty
}

Jong Sook Yi

Dr. Jin's Premium Nose Clinic, Seoul 06524, South Korea.

Correspondence to: Dr. Jong-Sook Yi, Dr. Jin's Premium Nose Clinic, Seocho-gu, gangnamdaero, Seoul 06524, South Korea. E-mail: jjongmari@nate.com

How to cite this article: Yi JS. Preoperative considerations, operative preparation, and postoperative carefor rib cartilage use in rhinoplasty. Plast Aesthet Res 2019;6:7. http://dx.doi.org/10.20517/2347-9264.2018.77

Received: 12 Nov 2018 First Decision: 19 Nov 2018 Revised: 4 Mar 2019 Accepted: 15 Mar 2019 Published: 26 Mar 2019

Science Editor: Hong Ryul Jin Copy Editor: Cai-Hong Wang Production Editor: Huan-Liang Wu

\begin{abstract}
Rib cartilage is the most reliable material for structural support and dorsal augmentation in Asian rhinoplasty with its robust strength and bountiful amount. Its value is incomparable especially in complex, cartilage-depleted revision surgery or major reconstruction. There are many articles regarding harvesting and carving of rib cartilage in rhinoplasty, however, only few has focused on preoperative and postoperative issues. Preoperatively, evaluating cartilage availability, assessing quality and quantity of cartilage, and choosing the cartilage to harvest are necessary. Although easily overlooked, proper postoperative management of rib cartilage rhinoplasty patients is key to prevent infection and heighten patient satisfaction. Here in, I would like to introduce how I evaluate rib cartilage rhinoplasty patients preoperatively and manage them postoperatively to maximize the surgical results.
\end{abstract}

Keywords: Rib cartilage, rhinoplasty, autologous graft, preoperative evaluation, postoperative management

\section{INTRODUCTION}

Cartilage grafts are widely used in nasal surgery. Although septal and auricular cartilage are easy to harvest, they often lack the amount necessary for many situations like revision rhinoplasty, severe deformity or trauma. As such, a surgeon is often compelled to use other sources ${ }^{[1,2]}$. Rib cartilage is the most reliable material for structural support and augmentation with its robust strength and bountiful amount. It can

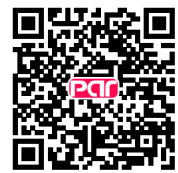


provide all of the necessary grafts from a single donor site with fewer complications ${ }^{[1]}$. However, many novice surgeons in rhinoplasty who are not familiar with chest anatomy, have difficulty in initiating the use of rib cartilage. The essential guidelines for use of rib cartilage in rhinoplasty are safe harvesting, and effective and appropriate carving of the rib cartilage. There are also several important factors to consider in a preoperative consultation for rib cartilage rhinoplasty. These considerations include an assessment of cartilage availability, and the choice of cartilage for harvesting. In addition, postoperative management is also important for optimal results. Herein, we introduce preoperative evaluation for assessment of proper rib cartilage and postoperative care.

\section{PREOPERATIVE EVALUATIONS}

\section{Decision to use a rib: consideration factors}

When the decision is made that rib cartilage is required, the first step is to check its availability. According to previous studies, sex and age are best related to the quality of rib cartilage ${ }^{[1-5]}$. In general, younger patients have more flexible and softer cartilage than older persons ${ }^{[1-5]}$. Contrary to our expectations, however, surgeons often encounter severely calcified rib cartilage, especially in young females. Sunwoo et al.$^{[6]}$ reported that $22.5 \%$ of teenage female patients showed calcification, and as early as 14 years old in some, which can suggest that the onset of rib cartilage calcification is earlier in women than in $\operatorname{men}^{[6,7]}$. Calcification makes it difficult not only to perform the graft manipulation, but also makes it more difficult to predict outcome because of its irregular absorption ${ }^{[7]}$. In addition, the risk of donor site morbidity may increase if the rib cartilage is severely calcified ${ }^{[6]}$. Therefore, preoperative assessment of the calcification degree by obtaining a series of radiographs of the rib or by pricking the rib cartilage with a fine needle are necessary regardless of a patient's sex or age [Figure 1]. Computed tomographic (CT) scans of chest are best used to specify calcification pattern with overall features of rib in many previous studies ${ }^{[5-8]}$. However, CT is more expansive and the radiation exposure is higher than simple X-ray. In my practice, simple rib X-ray provides enough information regarding the degree of calcification and size and shape of cartilage, thus, enables me to judge its availability in rhinoplasty safely with relatively low cost. Luckily, not all calcified cartilages are contraindication for harvest. Grades of cartilage calcification can be classified by the percentage of calcified lesion, and more than $25 \%$ can be regarded as meaningful calcification ${ }^{[6]}$. A mild degree $(<25 \%)$, marginal type calcification (calcification along the periphery of rib cartilage) is often acceptable, but a central/granular type with moderate calcification $(>25 \%)$ is not suitable in most cases ${ }^{[6]}$.

In selection of an appropriate amount of rib cartilage, costal cartilage is harvested from the sixth through eighth ribs according to its shape and purposes ${ }^{[1,6,8]}$. Right side cartilages are commonly preferred than the left to avoid injury to the pericardium and confusion of postoperative chest pain from angina $a^{[1-9]}$. Interestingly, however, a study revealed that the greatest amount of costal cartilage from the sixth, seventh, and eighth rib was identified on the left side than right on CT scan data ${ }^{[10]}$. Therefore, rib cartilage harvesting from the left side can be a viable option in those patients who have had previous rib surgery, radiotherapy, or trauma with destruction of costal cartilage on the right side ${ }^{[10]}$.

The sixth rib is usually at an ideal depth and the width is wide. But the straight piece is shorter than the seventh rib and has slight genu. Furthermore, if the patient has a history of breast implant, care should be taken not to injure implant material since the sixth rib is typically directly located under an implant ${ }^{[1,6,8]}$. Seventh rib cartilage has been known as the safest anatomically. It is situated over the abdominal cavity and thus can lessen the chance of pneumothorax since the parietal pleura of the lung runs down to reach the lower edge of the sixth rib at the xyphoid-chondral junction to the upper edge of the ninth rib cartilage laterally. The internal thoracic artery and vein also descend medial to the ribs from the seventh rib and therefore vascular injury is rare ${ }^{[8]}$. Its contour is also suitable for any rhinoplasty grafts because of an appropriate length and width. Thus, the seventh rib from the right side appears to be most advisable for 

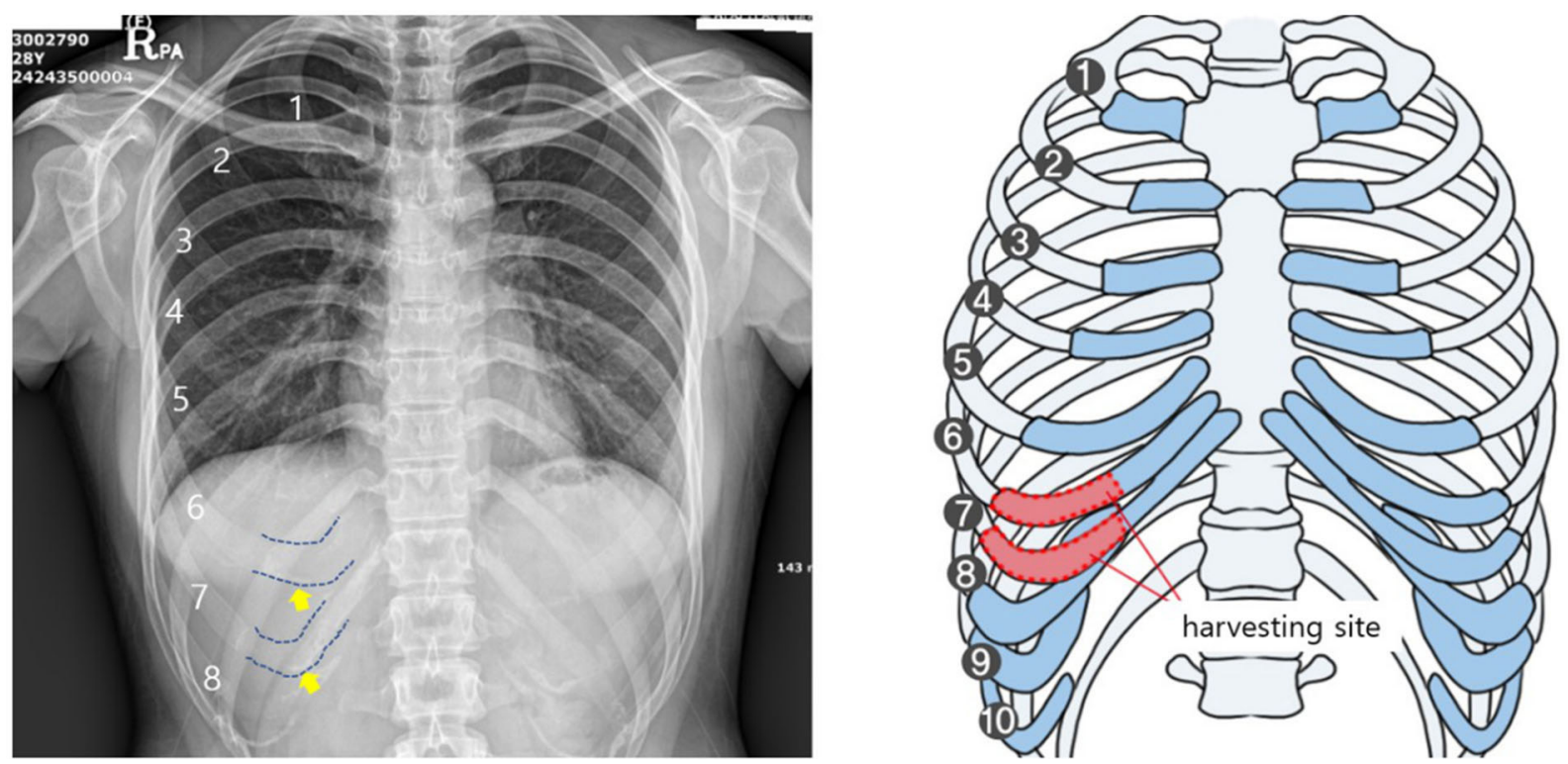

Figure 1. Appropriate number of rib cartilages are assessed on a simple rib series $\mathrm{X}$-ray. Shape, curvature, width and calcification can be reviewed. Dotted lines show curvature, shape, and width of rib cartilages. Yellow arrow shows linear marginal calcification on the 6th rib while granular type on the 7th

rhinoplasty in general ${ }^{[10]}$. The eighth rib has a significant connection with the adjacent rib and the width is narrow, which is inadequate for a dorsal graft ${ }^{[1,6,8,11]}$. Also, some senior physicians prefer the eleventh and twelfth free-floating ribs as graft material because they are naturally straight, require less carving and undergo less warping. Moreover, they are thin and easily accessible ${ }^{[12]}$.

Diced cartilage in temporalis fascia is an alternative choice when using rib cartilage if the length of the rib cartilage is insufficient as a one block dorsal graft or if the patient has very thin skin. In a case with a spotty calcified rib, it would be also better to dice for major dorsal augmentation to prevent unpredictable absorption $^{[13]}$.

\section{OPERATIVE PREPARATION}

Identify selected rib cartilage from surface anatomy: stepwise approach

After selecting an ideal rib from a rib series or CT scan, the next step is to find an exact rib from the chest surface with manual palpation. Fortunately, prior studies from cadaver and CT scans show that sex, age and ethnic background had little effect on determining costal cartilage anatomy ${ }^{[1,1]}$. For this process, the clavicle and xyphoid process are first marked on the skin in a supine position, and then the number of ribs is counted serially along the lateral rib cage [Figure 2]. The first palpable rib cartilage just below the clavicle is usually the second rib. In patients with a breast implant or that are obese, it is more helpful to count the ribs at the medial side rather than lateral. In a very difficult patient, the eighth rib can be found from the transverse plane passing through spinous process of the T12 vertebra in a lateral position ${ }^{[11]}$.

Over the chosen rib cartilage, the osseocartilagenous junction can be localized by pricking the rib using a 26-27 gauge needle considering its contour ${ }^{[1,2,6]}$. Harvesting rib cartilage from the osseocartilaginous junction can provide a maximal straight piece and volume with limited incision ${ }^{[11]}$. While pricking, great caution should be taken not to poke the pleura or lung parenchyma, resulting in a closed tension pneumothorax. For novices, it is recommendable to grab the superior and inferior margin of the selected rib with the other index and middle fingers to assure a midline [Figure 3]. After marking the osseocartilagenous junction, a horizontal skin incision is started from the marking and moving medially along the skin crease [Figure 4]. 


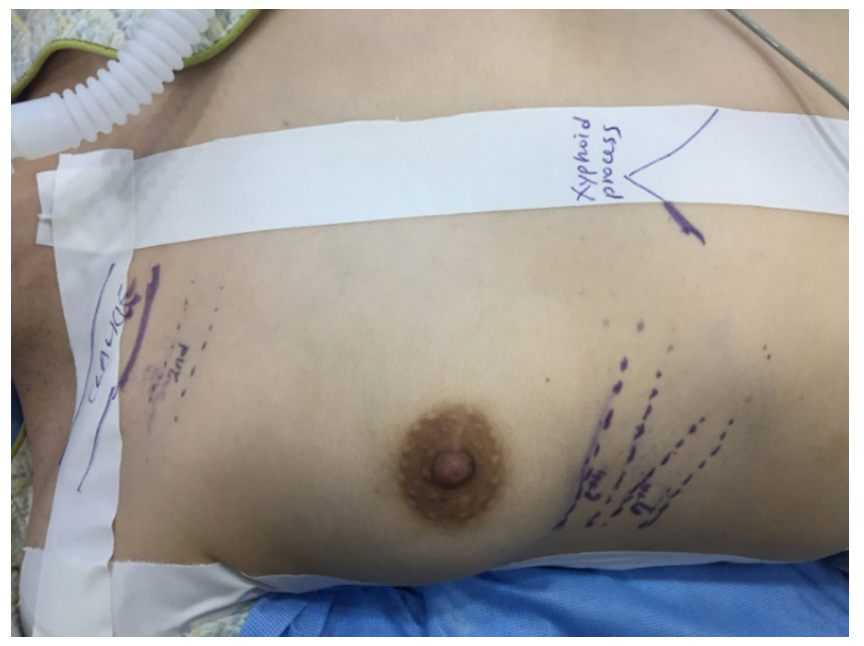

Figure 2. Rib cartilages are identified on supine position. The clavicle and xyphoid process is a key landmark and the first rib below the clavicle is the second rib

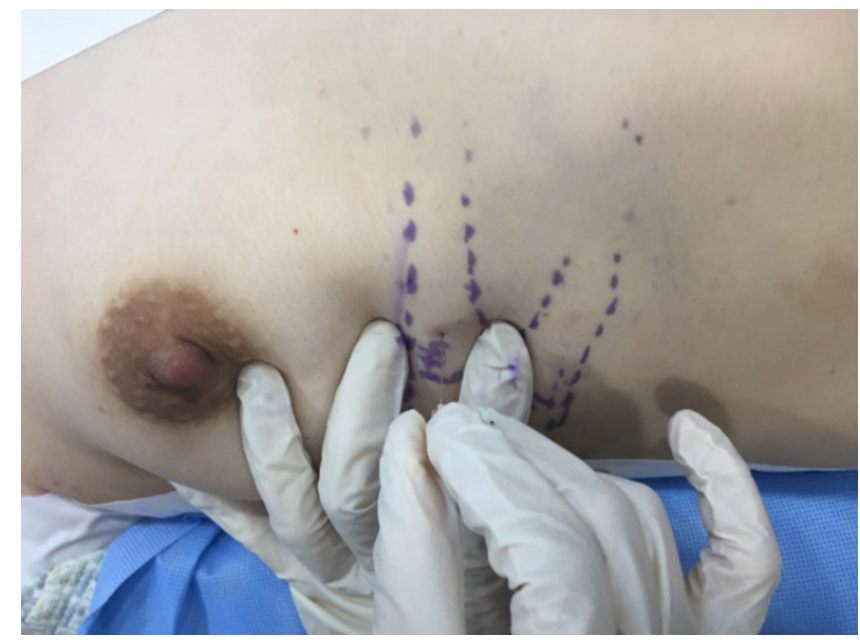

Figure 3. While needle pricking, keeping a midline is crucial to avoid tension pneumothorax. Grabbing selected ribs with two fingers from the other hand are helpful to guide the right direction

\section{POSTOPERATIVE CARE}

After wound closure, routine postoperative chest X-ray can be recommended until the surgeon is familiar with the procedure ${ }^{[1]}$. The chest wound is compressed lightly with a bandage and kept for 3 days to prevent hematoma. Drain is unnecessary in most cases. Pain is the most common complaint after costal cartilage harvest, regardless of purpose or extent. Many authors reported that donor site pain usually peaked in the first week and diminished slowly over 3 months ${ }^{[13-19]}$. In the past, local application of a long-lasting anesthetic substance close to the intercostal nerve was used to reduce extensive postoperative pain ${ }^{[15,16]}$. Anantanarayanan et al. ${ }^{[16]}$ reported that the use of catheter-based ropivacaine provided an earlier return to normal function with significant long duration, hence, decreasing the need for rescue analgesics. Recently, however, other studies have proven that severe pain can be minimized with modification of rib harvest techniques. For example, after superior and inferior perichondrium elevation, special precaution is taken not to injure the inferior line neurovascular bundle. Rib harvesting can also be limited to the outer lamellar while preserving the internal costal arch. By preserving the inner lamellar of the rib, postoperative morbidities, including pain, splinting and pneumothorax, can be reduced ${ }^{[17]}$. Recently, a muscle sparing 


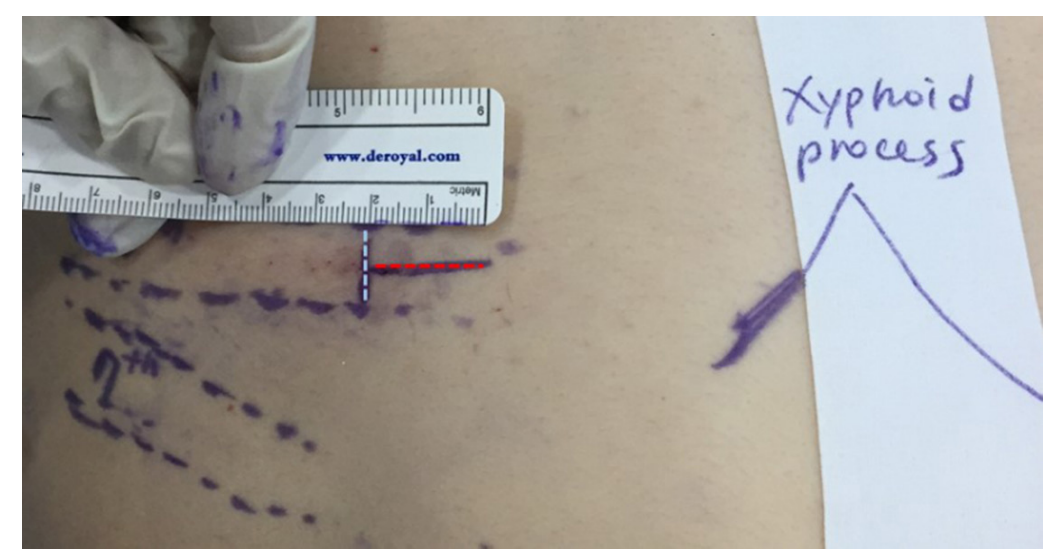

Figure 4. About a $2 \mathrm{~cm}$ transverse incision is made along the skin crease. The incision line (red dotted line) starts from the osseocartilaginous junction (blue dotted line) to obtain a maximal length in the piece of harvested cartilage

technique has been increasingly applied to reduce donor site discomfort ${ }^{[19,20]}$. Instead of transection, a blunt dissection of the external oblique muscle can significantly reduce pain during rest and movement. Thus, some authors recommend routine use of a muscle-sparing technique in autologous costal cartilage harvesting ${ }^{[20]}$. With these procedures, regular pain pills are sufficient to control postoperative chest pain. The author usually prescribes routine antibiotics and oral pain pills for a week after surgery with this muscle sparing technique. The pain tends to be peaked in 3-4 days and diminishes slowly over a week. Long lasting chest pain is a rare entity after rib cartilage harvest, however, long-lasting pain even months after surgery is possible especially when multiple rib cartilages were harvested. Even with a little pain after harvest of rib cartilage, restriction of activity is not recommended and rather light daily activities like walking, sitting are recommended from the day after surgery. However, more heavy activities like running, weight training, or playing with instruments are recommended to resume in 3 weeks.

Scar management is also an important part of postoperative care. To minimize unwanted skin abrasion, unidirectional skin retraction is helpful during harvest to prevent skin margin abrasion. The wound is closed in 4 layers; muscle, fascia, subcutaneous tissue and skin. Multilayered sutures are helpful to reduce vertical tension from stretch of upper body. Antibiotic ointment is put to incision scar once a daily for a week. A patient with a history of keloids or hypertrophic scar, triamcinolone can be injected at the costal cartilage harvest site as a preventative measure ${ }^{[1]}$. Wound stitch-out is performed in a week and then silicone scar sheets can be applied for two months to reduce visible scars. The author experienced elevated scar flattening after triamcinolone injection twice at two weeks interval at the donor site scar and the results were often acceptable in most patients. Yang et al. ${ }^{[21]}$ compared a VAS cosmetic score of costal cartilage harvest site scar from a retroauricular skin incision scar and reported that there was no significant difference at 6 months post operation.

Infection prevention needs special attention when heavy amount of rib cartilage was used in multiply revised patients. They tend to have poor blood supply, often the skin is stretched more, and the septum is also explored which all contribute to raise the infection chance. In this case, I give them IV antibiotics for a few more days after surgery and pay special attention to the intranasal hygiene to prevent retrograde infection from the nasal cavity. Everyday dressing of the nasal cavity and the wound with betadine solution and antibiotic ointment for a week is recommended. I teach patients to soak the nasal cavity often with betadine gauze for a week at home. After casting and stitch at one week postoperatively, I see them once again after a week to check any sign of infection and tell them to pay special attention to any sign of swelling, redness, and pain of the nose till follow-up time. 


\section{CONCLUSION}

To get optimal result in using rib cartilage, thorough preoperative evaluation and proper postoperative care should be achieved. The author introduced how to seek and select appropriate rib cartilage from chest surface and radiographic images. Pain control, scar management, and infection prevention are three major consideration factors in postoperative care and can be managed well with advanced techniques.

\section{DECLARATIONS}

\section{Authors' contributions}

The author contributed solely to the article.

\section{Availability of data and materials}

Not applicable.

\section{Financial support and sponsorship}

None.

\section{Conflicts of interest}

The author declared that there are no conflicts of interest.

\section{Ethical approval and consent to participate}

Not applicable.

\section{Consent to publication}

Not applicable.

\section{Copyright}

(c) The Author(s) 2019.

\section{REFERENCES}

1. Chung V, Toriumi DM. The use of costal cartilage for dorsal augmentation and tip grafting. Aesthetic Plastic Surgery of the East Asian Face. Thieme Medical Publishers; 2016. pp. 26-47.

2. Park JH, Jin HR. Use of autologous costal cartilage in Asian rhinoplasty. Plast Recostr Surg 2012;130:1338-48.

3. McCormick WF, Stewart JH. Ossification patterns of costal cartilages as an indicator of sex. Arch Pathol Lab Med 1983;107:206-10.

4. Navani S, Shah JR, Levy PS. Determination of sex by costal cartilage calcification. Am J Roentgenol Radium Ther Nucl Med 1970;108:771-4.

5. Sanders CF. Sexing by costal cartilage calcification. Br J Radiol 1966;39:233.

6. Sunwoo WS, Choi HG, Kim DW, Jin HR. Characteristics of rib cartilage calcification in Asian patients. JAMA Facial Plast Surg 2014;16:102-6.

7. Elkeles A. Sex differences in the calcification of the costal cartilages. J Am Geriatr Soc 1966;14:456-62.

8. Jung DH, Choi SH, Moon HJ, Chung IH, Im JH, et al. A cadaveric analysis of the ideal costal cartilage graft for Asian rhinoplasty. Plast Reconstr Surg 2004;114:545-50.

9. Wustrow TP, Kastenbauer E. Surgery of the internal nasal valve. Facial Plast Surg 1995;11:213-27.

10. JP Windfuhr, Chen YS, Güldner C, Neukirch D. Rib cartilage harvesting in rhinoplasty procedures based on CT radiological data. Acta Otolaryngol 2011;131:67-71.

11. Lepage D, Tatu L, Loisel F, Rey PB, Obert L, et al. Anatomical and computed tomography study of the eight costochondral junctions: topography for costochondral graft harvesting. Surg Radiol Anat 2016;38:809-15.

12. Moretti A, Sciuto S. Rib grafts in septorhinoplasty. Acta Otorhinolaryngol Ital 2013;33:190-5.

13. Park P, Jin HR. Diced cartilage in fascia for major nasal dorsal augmentation in Asians: a review of 15 consecutive cases. Aesthet Plast Surg 2016;40:832-9.

14. Uppal RS, Sabbagh W, Chana J, Gault DT. Donor-site morbidity after autologous costal cartilage harvest in ear reconstruction and approaches to reducing donor-site contour deformity. Plast Reconstr Surg 2008;1216:1949-55.

15. Rasp G, Staudenmaier R, Ledderose H, Kastenbauer E. Autologous rib cartilage harvesting: operative procedure and postoperative pain reduction. Laryngorhinootologie 2000;79:155-9. 
16. Anantanarayanan P, Raja DK, Kumar JN, Sneha P, Christabel A, et al. Catheter-based donor site analgesia after rib grafting: a prospective, randomized, double-blinded clinical trial comparing ropivacaine and bupivacaine. J Oral Maxillofac Surg 2013;71:29-34.

17. Yilmaz M, Vayvada H, Menderes A, Mola F, Atabey A. Dorsal nasal augmentation with rib cartilage graft: long-term results and patient satisfaction. J Craniofac Surg 2007;18:1457-62.

18. Nelson M, Gaball C. Technique to reduce time, pain, and risk costal cartilage harvest. JAMA Facial Plast Surg 2017;19:333-4.

19. Fedok FG. Costal cartilage grafts in rhinoplasty. Clin Plast Surg 2016;43:201-12.

20. Özücer B, Dinç ME, Paltura C, Koçak I, Dizdar D, et al. Association of autologous costal cartilage harvesting technique with donor-site pain in patients undergoing rhinoplasty. JAMA Facial Plast Surg 2018;20:136-40.

21. Yang HC, Cho HH, Jo JY, Jang CH, Cho YB. Donor-site morbidity following minimally invasive costal cartilage harvest technique. Clin Exp Otolaryngol 2015;8:13-9. 